\title{
Size and molecular flexibility affect the binding of ellagitannins to bovine serum albumin
}

Article

Accepted Version

Dobreva, M., Green, R.J., Mueller-Harvey, I., Salminen, J.-P., Howlin, B.J. and Frazier, R. (2014) Size and molecular flexibility affect the binding of ellagitannins to bovine serum albumin. Journal of Agricultural and Food Chemistry, 62 (37). pp. 9186-9194. ISSN 0021-8561 doi:

https://doi.org/10.1021/jf502174r Available at https://centaur.reading.ac.uk/37617/

It is advisable to refer to the publisher's version if you intend to cite from the work. See Guidance on citing.

To link to this article DOI: http://dx.doi.org/10.1021/jf502174r

Publisher: American Chemical Society

All outputs in CentAUR are protected by Intellectual Property Rights law, including copyright law. Copyright and IPR is retained by the creators or other copyright holders. Terms and conditions for use of this material are defined in the End User Agreement. 


\section{CentAUR}

Central Archive at the University of Reading

Reading's research outputs online 
Size and Molecular Flexibility affect the Binding of Ellagitannins to Bovine Serum Albumin

Marina A. Dobreva, ${ }^{\dagger}$ Rebecca J. Green, ${ }^{*},+$ Irene Mueller-Harvey, ${ }^{*},{ }^{\dagger}$ Juha-Pekka Salminen, ${ }^{\S}$ Brendan J. Howlin, ${ }^{\#}$ Richard A. Frazier ${ }^{\ddagger}$

${ }^{\dagger}$ School of Agriculture, Policy and Development, University of Reading, Earley Gate, PO Box 236, Reading, RG6 6AT, UK;

${ }^{\ddagger}$ School of Chemistry, Food and Pharmacy, University of Reading, Whiteknights, PO Box 224, Reading, RG6 6AP, UK;

${ }^{\S}$ Department of Chemistry, University of Turku, Vatselankatu 2, Turun Yliopisto, Turku, FI-20014, Finland.

${ }^{\#}$ Chemistry Department, FEPS, University of Surrey, Guildford, Surrey, GU2 7XH, UK.

\section{AUTHOR INFORMATION:}

*Phone: +44-118-378-8446. Fax: +44-118-378-6562.

E-mail: rebecca.green@reading.ac.uk.

*Phone: +44-118-378-6619. Fax: +44-118-935-2421.

E-mail: i.mueller-harvey@ reading.ac.uk. 
1 ABSTRACT: Binding to bovine serum albumin of monomeric (vescalagin and

2 pedunculagin) and dimeric ellagitannins (roburin A, oenothein B and gemin A) was

3 investigated by isothermal titration calorimetry and fluorescence spectroscopy, which

$4 \quad$ indicated two types of binding sites. Stronger and more specific sites exhibited

5 affinity constants, $K_{l}$, of $10^{4}$ to $10^{6} \mathrm{M}^{-1}$ and stoichiometries, $n_{l}$, of 2 to 13 and

6 dominated at low tannin concentrations. Weaker and less-specific binding sites had $K_{2}$

7 -constants of $10^{3}$ to $10^{5} \mathrm{M}^{-1}$ and stoichiometries, $n_{2}$, of 16 to 30 , and dominated at

8 higher tannin concentrations. Binding to stronger sites appeared dependent on tannin

9 flexibility and presence of free galloyl groups. Positive entropies for all but gemin A

10 indicated that hydrophobic interactions dominated during complexation. This was

11 supported by an exponential relationship between the affinity, $K_{1}$, and the modeled,

12 hydrophobic accessible surface area and by a linear relationship between $K_{l}$ and the

13 Stern-Volmer quenching constant, $K_{S V}$.

14

KEYWORDS: ellagitannins, isothermal titration calorimetry, fluorescence, molecular flexibility, molecular size, hydrophobic accessible surface area 


\section{INTRODUCTION}

Many plants, herbal drugs and foods contain not only the widely-studied condensed tannins but also ellagitannins (ETs). ${ }^{1,2}$ In fact, ellagitannins are much more common than previously recognized (Salminen - unpublished data). We consume ellagitannins in nuts, berries, fruit juices and wines ${ }^{3}$ and interest is growing in the medicinal and health effects of these polyphenols. ${ }^{3-8}$

Dietary polyphenols may prevent diseases through their ability to quench free radicals, which are generated under oxidative stress. ${ }^{3}$ Whilst ETs that contain hexahydroxydiphenoyl (HHDP) groups are rapidly metabolized into ellagic acid and urolithins after consumption, C-glucosidic ETs contain a nonahydroxytriphenoyl (NHTP) group and are difficult to hydrolyse. ${ }^{5}$ Pedunculagin and gemin A are examples of HHDP-containing ETs and vescalagin and roburin A contain NHTP group(s) (Figure 1). Many ETs have high water solubility and surprisingly high bioavailability. ${ }^{9}$ They are also capable of interacting with various molecular targets that affect signaling pathways ${ }^{10}$ and can inhibit tumor promotion. ${ }^{11}$ For example vescalagin enters cells rapidly, ${ }^{4}$ strongly inhibits DNA topoisomerase ${ }^{12-14}$ and interacts selectively with actin filaments. ${ }^{5}$ Oenothein B is one of the most active ETs in terms of host-mediated antitumor activity and generates an immune response by stimulating interleukin 1 production. ${ }^{1}$ Oenothein B and ET metabolites such as ellagic acid and urolithins can also influence histone acetylation/deacetylation and thus inflammatory responses, ${ }^{15}$ which play an important role in the development of agerelated diseases. 
43 These and many other examples illustrate that tannins are not just non-specific protein-precipitating agents but can also be involved in specific ${ }^{16}$ and targeted interactions with certain amino acids. ${ }^{17}$ Tannin-protein interactions have been investigated by a wide range of physico-chemical techniques and were summarized previously. ${ }^{5,18,19}$ These included competitive binding assays, NMR spectroscopy, circular dichroism, mass spectrometry, infra-red spectroscopy, dynamic light scattering, small angle X-ray scattering, transmission electron microscopy, equilibrium dialysis, nephelometry, calorimetry, fluorescent quenching, ion mobility, ${ }^{20}$ and surface plasmon resonance. ${ }^{21}$ Isothermal titration calorimetry (ITC) provides information not only on thermodynamic parameters and the strengths of the tannin-protein interaction but also on the stoichiometry of the resulting complex. Other work has shown that both hydrophobic effects ${ }^{5}$ and hydrogen bonding are involved. ${ }^{16,22}$ Which of these is the dominant factor depends on the precise polyphenol/protein interactions and experimental conditions. ${ }^{5,23}$ Both the flexibility and size of the tannins and proteins appear to affect these interactions. ${ }^{16,24,25}$ When gallotannin (GT)- and ET-binding to gelatin and BSA were compared, it was found that equilibrium binding constants for flexible GTs with the globular BSA were between $10^{4}$ to $10^{5} \mathrm{M}^{-1}$ and for ETs interacting with the highly flexible gelatin these were ca. $10^{6} \mathrm{M}^{-1} \cdot{ }^{19}$

ETs are unique amongst tannins as i) they can be isolated as pure compounds (unlike most condensed tannins, which tend to be obtained as closely related mixtures); ii) they possess a wide range of molecular flexibilities (in contrast to gallotannins or condensed tannins); and iii) their water solubilities are inversely correlated to their protein precipitation capacities. ${ }^{9}$ ETs, therefore, offer an opportunity for investigating 
the relative importance of molecular flexibility and size within a series of matched tannin compounds.

We chose two monomeric and three dimeric ETs that differ in molecular flexibility (i.e. their bond rotational flexibility) and water solubility. Vescalagin and its dimer, roburin A, represent ETs with NHTP groups that are linked to an acyclic glucose. They are consumed in wine that has been stored in oak barrels. Walnuts are a rich source of pedunculagin, which represents ETs with HHDP groups and a cyclic glucose. The dimers, oenothein B and gemin A can be found in several European and Japanese herbal medicines ${ }^{1,26}$ and differ in their molecular flexibilities. The water solubilities of these ETs decrease in the following order: vescalagin $>$ pedunculagin $\approx$ roburin $\mathrm{A}>$ oenothein $\mathrm{B}>$ gemin $\mathrm{A} .{ }^{9}$

The present study investigated the effects of flexibility and size on the thermodynamics of the ET-protein interaction using isothermal titration calorimetry and fluorescence spectroscopy to probe their interaction mechanisms and binding sites. We choose bovine serum albumin (BSA) as the protein-binding partner for two reasons. Firstly, BSA is a widely used model for globular proteins such as Rubisco, which is a major dietary protein. ${ }^{27,28}$ Recent studies have shown that the binding affinity in tannin-protein complexes was negatively correlated with digestibility. ${ }^{29-31}$ Secondly, albumins are involved in transporting dietary polyphenols ${ }^{32,33}$ and drugs to their molecular targets, ${ }^{34,35}$ and exhibit a large sequence homology, e.g. $76 \%$ in the case of BSA and human serum albumin. ${ }^{36}$ It is, therefore, important to understand how ellagitannins interact with globular proteins, as this may also impact on proteindrug interactions in the digestive tract and in the blood plasma. ${ }^{35}$ 
MATERIALS AND METHODS

95

Ellagitannins. Two monomeric ellagitannins (pedunculagin and vescalagin) and three dimeric ellagitannins (oenothein B, roburin A and gemin A) were extracted from plants and purified as described previously. ${ }^{37}$

Isothermal Titration Calorimetry. Titrations of BSA (purity $\geq 99 \%$, essentially globulin free, 66 kDa; Sigma, Poole, Dorset, U.K.) with ellagitannins were performed using a NanoITC instrument (TA Instruments Ltd., Crawley, West Sussex, U.K.) as described previously ${ }^{19,28}$ with few adaptations. All solutions were prepared in $50 \mathrm{mM}$ citrate buffer at pH 6 and were degassed under vacuum prior to use. In a typical experiment, buffered BSA solution $(20 \mu \mathrm{M})$ was placed in the $950 \mu \mathrm{L}$ sample cell of the calorimeter and buffered ellagitannin solution $\left(3 \mathrm{mg} \mathrm{mL}^{-1}\right)$ was loaded into the injection syringe. Ellagitannin was titrated into the sample cell at $298 \mathrm{~K}$ as a sequence of 24 injections of $10 \mu \mathrm{L}$ aliquots for monomeric ellagitannins (pedunculagin and vescalagin) and 48 injections of $10 \mu \mathrm{L}$ aliquots for dimeric ellagitannins (oenothein $\mathrm{B}$, roburin A and gemin A). The time delay (to allow equilibration) between successive injections was $360 \mathrm{~s}$. The contents of the sample cell were stirred throughout the experiment to ensure thorough mixing.

Raw data were obtained as plots of heat $(\mu \mathrm{J})$ against injection number and featured a series of peaks for each injection. These raw data peaks were transformed using the instrument software to obtain a plot of observed enthalpy change per mole of injectant $\left(\Delta H_{\mathrm{obs}}, \mathrm{kJ} \mathrm{mol}^{-1}\right)$ against molar ratio (see ITC Data Analysis). 
Control experiments included the titration of buffered ellagitannin solutions into buffer, buffer into BSA and buffer into buffer; controls were repeated for each BSA concentration. Control experiments of buffer titrated into buffer or protein solutions both resulted in small and equal enthalpy changes for each successive injection of buffer and, therefore, were not further considered in the data analysis. ${ }^{38}$ Experimental data were analyzed after subtraction of the tannin into buffer control data from the sample data. Ellagitannin molecules tend to self-associate into aggregates due to hydrophobic groups; therefore, when injected from the syringe into buffer the ellagitannin molecules undergo an endothermic process of deaggregation, analogous to surfactant demicellization. ${ }^{39}$ The extent of deaggregation depends inversely on the concentration of ellagitannin already present in the sample cell; therefore, successive injections of ellagitannin into buffer lead to the observation of progressively lower endothermic enthalpy changes as has been illustrated in earlier work. ${ }^{39}$ The data are shown after subtraction of the effects of ellagitannin deaggregation, which means that the assumption is made that ellagitannins dissociate prior to binding.

ITC Data Analysis. Estimated binding parameters were obtained from ITC data using the Bindworks ${ }^{\mathrm{TM}}$ (Version 3.1.13, Applied Thermodynamics, Hunt Valley, MD, U.S.A.) data analysis program. Data fits were obtained using a model for two independent sets of multiple binding sites. For this model, the analytical solution for the total heat measured $(Q)$ is determined by the formula:

$$
Q=V[\mathrm{M}]\left\{\frac{n_{1} \Delta H_{1} K_{1}[\mathrm{~L}]}{1+K_{1}[\mathrm{~L}]}+\frac{n_{2} \Delta H_{2} K_{2}[\mathrm{~L}]}{1+K_{2}[\mathrm{~L}]}\right\}
$$

where $n_{1}$ and $n_{2}$ are the molar ratios of interacting species, $\Delta H_{1}$ and $\Delta H_{2}$ are the enthalpies, and $K_{1}$ and $K_{2}$ are the equilibrium binding constants (syn. affinity 
constants) for each of the two sets of multiple binding sites. ${ }^{40}$ The quality of fit was determined by calculation of $\chi^{2}$; the data fits were acceptable in each case since the $\chi^{2}$ values were less than the critical values for the appropriate degree of freedom.

Tryptophan Fluorescence Quenching. Fluorescence intensity was recorded using an FP-6200 spectrofluorimeter (JASCO UK Ltd., Great Dunmow, Essex, U.K.) with selective excitation of tryptophan residues in BSA at $295 \mathrm{~nm}$. The excitation and emission slits were $5 \mathrm{~nm}$ and the emission spectrum was recorded between 300 and $500 \mathrm{~nm}$. The measured fluorescence intensities are reported without correction for inner filter effects. $^{41}$

Titration of BSA $(5 \mu \mathrm{M}, 2 \mathrm{~mL})$ with ellagitannin $\left(3 \mathrm{mg} \mathrm{mL}^{-1}, 500 \mu \mathrm{L}\right)$ was carried out as a sequence of aliquots ( $10 \mu \mathrm{L}$ up to volume of $100 \mu \mathrm{L}, 20 \mu \mathrm{L}$ up to a volume of $460 \mu \mathrm{L})$. For the calculation of quenching constants, the data were plotted as a SternVolmer plot of $F_{0} / F$ against $[Q]$ and the quenching constant, $K_{S V}$, calculated by linear regression. ${ }^{42}$ According to the Stern-Volmer equation:

$$
\frac{F_{0}}{F}=1+k_{q} \tau_{0}[Q]=1+K_{S V}[Q]
$$

where, $F_{0}$ and $F$ are the fluorescence intensities before and after the addition of the quencher, respectively, $k_{q}$ is the bimolecular quenching constant, $\tau_{0}$ is the lifetime of the fluorophore in the absence of quencher, $[Q]$ is the concentration of the quencher (in this case tannin) and $K_{S V}$ is the Stern-Volmer quenching constant.

\section{However, in the case of combined dynamic and static quenching, where the Stern-} Volmer plot exhibits an upward curvature (concave towards the $y$-axis) an alternative equation can be used for fitting: ${ }^{42}$ 
168 where,

$$
K_{a p p}=\left[\frac{F}{F_{o}}-1\right] \frac{1}{[Q]}=\left(K_{D}+K_{S}\right)+K_{D} K_{S}[Q]
$$

(Equation 4)

170 The actual plot of $\mathrm{K}_{\mathrm{app}}$ or $\left(\left(\mathrm{F} / \mathrm{F}_{\mathrm{o}}-1\right) / \mathrm{Q}\right)$ versus [Q] yields a straight line with an

171 intercept of $K_{D}+K_{S}$ and a slope of $K_{D} K_{S}$.

172

Molecular Modeling. Molecular modeling was performed with MOE2011.10 (Chemical Computing Ltd., Montreal, Canada). Atomistic models of the respective tannins were built from chemical structures on an atom by atom basis using the Builder Module of MOE. All models were energy minimized to convergence (rms gradient of $0.05 \mathrm{Kcal} / \mathrm{mol}$ ) using the MMX94x force field by smart minimization (steepest descents, followed by conjugate gradient and truncated Newton-Raphson methods). Physical parameters were calculated from these models using the QuasAR module in MOE. Parameters calculated were accessible surface area (ASA), hydrophobic accessible surface area (ASA_H). Molecular flexibility was calculated by performing a conformational energy search about the torsion angles of each model using Lowmode Molecular Dynamics in the Conformational Analysis module of MOE. Results from conformational analysis were stored in databases of conformations for each tannin indexed by final energy.

\section{RESULTS AND DISCUSSION}

This study investigated the interaction between BSA and five ellagitannin compounds using isothermal titration calorimetry and fluorescence spectroscopy. The molecular flexibility and surface areas of five ellagitannins and pentagalloyl glucose were 
modelled in order to improve our understanding of these interactions.

Molecular Flexibility of Ellagitannins. Molecular simulation was used to assess molecular flexibility in terms of numbers of low energy conformations located and energy of these conformers (Table 1). Modeling experiments ${ }^{43}$ demonstrated previously that the nonahydroxytriphenoyl (NHTP) group is much less flexible than the hexahydroxydiphenoyl (HHDP) group (Figure 1) and the low energy conformers of the monomers clearly reflect the extent of these structural constraints: pentagalloyl glucose conformers $\left(\mathrm{n}=21\right.$ ) ranged from $110-116 \mathrm{kcal} \mathrm{mol}^{-1}$ (no NHTP or HHDP groups), pedunculagin conformers $(\mathrm{n}=4)$ from 158-164 $\mathrm{kcal} \mathrm{mol}^{-1}$ (2 HHDP groups) and vescalagin conformers $(\mathrm{n}=18)$ from 229 to $236 \mathrm{kcal} \mathrm{mol}^{-1}$ (1 NHTP, 1 HHDP group). The low energy conformers of the dimers followed a similar trend: oenothein B conformers $(\mathrm{n}=2)$ had 278-280 $\mathrm{kcal} \mathrm{mol}^{-1}$ (2 HHDP groups), gemin A ( $\left.\mathrm{n}=1\right)$ had $323 \mathrm{kcal} \mathrm{mol}^{-1}$ (3 HHDP groups) and roburin A conformers (n=4) had 406-412 kcal mol $^{-1}$ (2 NHTP, 2 HHDP groups).

\section{Isothermal Titration Calorimetry of Ellagitannin-BSA Interactions and Data}

Fitting. ITC experiments for ellagitannin titration into BSA were carried out for five ellagitannins (depicted in Figure 1). Figure 2 shows the ITC binding isotherms for the interaction with BSA of vescalagin and its dimer, roburin A, consisting of the experimental data (as points) and the binding model that best fitted to each data set (as lines). For both ellagitannins, the binding interaction led to an exothermic response, which decreased in magnitude with successive titration injections and signifies a saturation of binding sites on BSA. 
216 As found in previous studies, ${ }^{24,39}$ small but significant exothermic peaks were

217 observed at higher tannin:protein molar ratios at and beyond the point of apparent

218 binding site saturation. This means that titration experiments did not reach zero

219 enthalpy change, even after removing effects from control experiments (e.g. tannin

220 titration into buffer). Therefore, we have assumed that the interaction between

221 ellagitannin and BSA has not reached completion at the higher ellagitannin:protein

222 ratios measured. As a consequence, upon fitting the data to a binding model, this long

223 tail of exothermic peaks prevents the use of a model based on a single set of multiple

224 binding sites (one-site model), and, therefore, all data were fitted to a two independent

225 binding site model. A previous study of the interaction between pentagalloyl glucose

226 with BSA or Rubisco described the selection of the two independent binding site

227 model in more detail. ${ }^{28}$

Figure 3 shows the ITC binding isotherms for pedunculagin and the related dimers

oenothein B and gemin A. As observed for the vescalagin and roburin A isotherms, the binding isotherm for the dimers shows a clear two-phase shape to the isotherm that signifies two or more binding sites are involved in the interaction. For the oenothein B and gemin A ellagitannins, the titration experiments were extended by increasing the number of titration injections to observe the interaction at higher molar ratios (see Methods for more details). The thermodynamic binding parameters for the ellagitannin-BSA interaction were obtained from a two-site model and are shown in Table 2, which also includes data from our previous study on pentagalloyl glucose binding to BSA for comparison. ${ }^{28}$ 
Molecular Flexibility. In these studies, the excellent fits of the ITC data have enabled us to probe the effects of different structural characteristics of the ellagitannins on their interaction with BSA. The results will be discussed in terms of monomer-dimer pairs and molecular flexibilities. The weakness of the second binding site allows, however, for some uncertainties in exact values for these fits, but does confirm our previous observations, which included related ellagitannins and suggested that these were able to bind (adsorb) non-selectively. ${ }^{24,28,39}$

The ellagitannin monomers, pendunculagin and vescalagin, show equivalent strengths of interaction $\left(K_{1}=4.2\right.$ and $\left.5.2 \times 10^{4} \mathrm{M}^{-1}\right)$ and so do the roburin $\mathrm{A}$ and oenothein $\mathrm{B}$ dimers $\left(K_{1}=2.3\right.$ and $\left.6.5 \times 10^{5} \mathrm{M}^{-1}\right)($ Table 2$)$. In contrast, the $K_{1}$ affinity constants of pentagalloyl glucose and gemin A were one order of magnitude larger than for the other monomers and dimers $\left(K_{1}=1.8 \times 10^{5}\right.$ and $1.8 \times 10^{6} \mathrm{M}^{-1}$, respectively). This coincides with the fact, as pointed out above that both have low conformational energies and are flexible molecules. Taken together gemin A has several features, i.e. single bonds and 'free' galloyl groups, which may allow selective binding to specific amino acids on the protein surface. These findings are in line with two other studies that reported weaker binding by vescalagin ${ }^{44}$ and by its isomer, castalagin, ${ }^{29}$ to BSA compared to pentagalloyl glucose. It appears that pentagalloyl glucose and gemin A have optimal structures for BSA binding within the group of tannins studied here.

For both binding sites the interaction in terms of the affinity constants, $K_{1}$ and $K_{2}$, is approximately one order of magnitude different between monomers and dimers (Table 2). For each of the comparable ellagitannin monomer-dimer pairs, the dimer was shown to bind more strongly than the monomer. It can also be seen that $K_{2}$ is one 
order of magnitude weaker than $K_{l}$. This weaker second binding site is likely to correspond to non-specific adsorption of tannins that may lead to coating of the protein surface $e^{28,45}$ and eventual precipitation of the tannin-protein complex in some systems. The binding isotherms in Figures 2 and 3 for the dimers show a greater exothermic response than for the corresponding monomers, e.g. roburin A $v s$ vescalagin $\left(\Delta H_{1}=-15.3 v s-7.5 \mathrm{~kJ} \mathrm{~mol}^{-1}\right)$, oenothein B $v s$ pedunculagin $\left(\Delta H_{1}=-21.6\right.$ $\left.v s-10.9 \mathrm{~kJ} \mathrm{~mol}^{-1}\right)$ and gemin A vs pentagalloyl glucose $\left(\Delta H_{1}=-47.4 v s-29 \mathrm{~kJ} \mathrm{~mol}^{-1}\right)$ (Table 2).

A closer look at the monomer series and the dimer series (each having tannins with similar molecular weights) suggests that the strength of binding is inversely related to the minimized energies and increases with a greater potential for rotational flexibility as follows (Table 1). The low energy conformers and binding constants, $K_{1}$, of the monomers are: $229-236 \mathrm{kcal} / \mathrm{mol}$ and $5.2 \times 10^{4} \mathrm{M}^{-1}$ for vescalagin, $158-164$ and $4.2 \times$ $10^{4}$ for pedunculagin, and $110-116$ and $1.8 \times 10^{5}$ for pentagalloyl glucose. For the dimer series the corresponding values are: $406-412 \mathrm{kcal} / \mathrm{mol}$ and $2.3 \times 10^{5} \mathrm{M}^{-1}$ for roburin A, 278-280 and $6.5 \times 10^{5}$ for oenothein B, 323 and $1.8 \times 10^{6}$ for gemin A.

\section{The number of strong (type 1) binding sites increases for ellagitannins with more} flexibility and 'free' galloyl groups from $n_{l}=1.9$ for roburin A to 4.1 for oenothein B and 13 for gemin A. The binding parameters for two of the monomer-dimer pairs, i.e. the vescalagin-roburin A and pedunculagin-oenothein B pairs, show similarities in the stoichiometry of the interaction. For the vescalagin-roburin A pair the stronger binding site $n_{1}$ is 2.5 for the monomer and 1.9 for the dimer; and for the second weaker binding site $n_{2}$ is 30 for the monomer and 16 for the dimer. For the 
pedunculagin-oenothein B pair $n_{1}$ is 2.1 for the monomer and 4.1 for the dimer; and $n_{2}$

292

293 is 30 for the monomer and 20 for the dimer. However, for the most flexible monomerdimer pair, which will be able to better support H-bonding, the stoichiometric values were much higher for the stronger binding site: $n_{1}$ was 26 for pentagalloyl glucose and 13 for gemin A. The difference reflects the fact that gemin A is twice the molecular size of pentagalloyl glucose. We reported previously that the sum of $n_{1}$ and $n_{2}$ indicated that 52 pentagalloyl glucose molecules are bound to BSA and this suggested surface coating as the BSA surface can theoretically accommodate 40 to 120 pentagalloyl glucose molecules. ${ }^{28}$ Similarly, the sum of $n_{1}$ and $n_{2}$ for gemin A indicated that 38 gemin A dimers were bound (which equates to 76 'monomers') and matches with the calculated surface area. It also suggests that gemin A due to its flexibility is capable of coating the whole BSA protein just like pentagalloyl glucose ${ }^{28}$ Comparable stoichiometries were found for commercial preparations of the flexible taratannins when assuming a molecular weight of 1500: i.e. 48:1 for the taratannin:BSA ratio. ${ }^{39}$

It is, however, of note that this molecular size effect was not observed to the same extent for the other pairs as monomers and dimers had similar $n_{1}$-values ( 2 to 4 ) and similar sums of $n_{1}+n_{2}$ (i.e. 33 for vescalagin/18 for roburin A and 32 for pedunculagin/24 for oenothein B). This indicates that the less flexible structures and especially roburin A were less able to stretch out on the BSA surface.

Many more binding constants are available for flavonoids and condensed tannins than for ellagitannins. Nevertheless, pure flavonoid compounds tend to have comparable affinities as the ellagitannins investigated here. $K_{a}$ constants with BSA of $10^{2}$ to $10^{5}$ 
$\mathbf{M}^{-1}$ were reported for monomeric and polymeric flavanols ${ }^{19,31}$ and of $10^{4}$ to $10^{5} \mathbf{M}^{-1}$

317 for flavones and flavonols. ${ }^{46-49}$ Examples of polyphenol complexes with human

318 serum albumin include epigallocatechin gallate and diosmetin interactions that

319 exhibited comparable Stern-Volmer affinity constants of $\sim 10^{5} \mathrm{M}^{-1}$ from fluorescence 320 data. $^{50-51}$

The findings also match results from previous studies, where less pure tannins had been used, and confirm that the tannin components of those fractions did indeed

324 dominate the interaction observed. ${ }^{19,24,39}$ The present study used a two-site binding 325 model and found $K_{1}$ of $10^{4}$ to $10^{5}$ and $K_{2}$ of $10^{3}$ to $10^{4} \mathrm{M}^{-1}$ for the monomers in BSA complexes. In comparison, data obtained with a single-site model gave $K$ values of

$327 \sim 10^{3} \mathrm{M}^{-1}$ for chestnut- and myrabolan-BSA complexes. ${ }^{24}$

329 Quideau et $a l^{5}$ reported that most polyphenols, including ellagitannins, when bound at 330 protein surfaces, exhibited affinity constants that were rarely above the micromolar 331 range, i.e. $K_{a}$ of $\leq 10^{6} \mathrm{M}^{-1}$. However, Xiao et al ${ }^{52}$ also reported much larger constants 332 of up to $10^{8} \mathrm{M}^{-1}$ for some flavanoid and flavonoid - human serum albumin complexes. 333 Not surprisingly, some receptor and enzyme targets had particularly high selectivities 334 with affinity constants at the subnanomolar scale, and varied between ellagitannins 335 even if these were of similar molecular weights. ${ }^{5,53}$

337 The data in Table 2 showed positive entropies at both types of binding sites for all but 338 one ellagitannin, i.e. gemin A. Positive $\Delta S_{1}$ values ranged from 33 to 65 and $\Delta S_{2}$ 339 values from 36 to $54 \mathrm{~J} \mathrm{~mol}^{-1} \mathrm{~K}^{-1}$ and are indicative of a dominance of hydrophobic 340 interactions. ${ }^{19,50,54}$ Gemin A was the only ellagitannin giving a negative entropy, $-39 \mathrm{~J}$ 
$\mathrm{mol}^{-1} \mathrm{~K}^{-1}$. For comparison purposes, we have included here also the entropies for pentagalloyl glucose, which shows that the highly flexible pentagalloyl glucose and gemin A had noticeably lower entropies at the stronger binding site than the other ellagitannins and were, therefore, apparently better able to form hydrogen bonds. ${ }^{54}$ The positive entropies for all ellagitannins at the second binding site are consistent with hydrophobic stacking on the BSA surface, although some uncertainty exists with these values as the affinities were very low for some tannins $\left(K_{2}=10^{3} \mathrm{M}^{-1}\right)$. The literature contains examples of both hydrogen bonding and hydrophobic interactions in tannin-protein complexes. ${ }^{16,19,22,50,55}$ Taken together, the present study also suggests that both types of bonding interactions occur in these ellagitannin-BSA complexes and slight structural differences appear to dictate, which of these will dominate.

\section{Quenching of the BSA Tryptophan Fluorescence by Ellagitannins. To}

complement the ITC measurements, ellagitannin binding to BSA was also investigated by fluorescence spectroscopy. These experiments measure the quenching of the intrinsic tryptophan fluorescence of $\mathrm{BSA}^{32}$ after binding with each of the tannins. Two tryptophan residues are found near the BSA surface, one is within its hydrophobic binding pocket (Trp 212) and the other within the surface region of the protein (Trp 134). ${ }^{49}$ Figure 4(a) shows Stern-Volmer plots that describe tryptophan quenching as a function of tannin concentration. In these plots $\mathrm{F}_{0} / \mathrm{F}$ increases as fluorescence intensity decreases in the presence of quencher $(F)$ relative to the native fluorescence in the absence of quencher $\left(\mathrm{F}_{0}\right)$. The data suggest that the rate of decrease in fluorescence with respect to quencher concentration depends on the molecular size and flexibility of the tannin. The data in Figure 4(a) revealed that four of the tannins fall into either monomer or dimer groups: the monomers, vescalagin 
and pedunculagin, exhibit similar quenching behavior, and the dimers, roburin A and oenothein B, also behave similarly to one another. The dimers were more efficient quenchers than the monomers, requiring approximately half the molar tannin concentration to produce equivalent levels of quenching. Indeed, if the quenching data are normalised, as in Figure 4(b) where Stern-Volmer plots are given as a function of 'tannin monomer' concentration, thus removing effects of molecular weight, it can be seen that related monomer-dimer pairs overlay, which demonstrates similar quenching efficiency per monomer unit. In contrast to these four tannins, the most flexible dimer, gemin $\mathrm{A}$, is a much more efficient quencher at much lower concentrations.

Pentagalloyl glucose is also a more efficient quencher, $K_{s v} \sim 2 \times 10^{6} \mathrm{M}^{-1},{ }^{28}$ than vescalagin and pedunculagin, but pentagalloyl glucose and gemin A are not a true monomer-dimer pair and thus do not overlay exactly.

The purpose of the Stern-Volmer plot of fluorescence quenching data is to generate a linearised data set that allows assessment of the ability of tannins to quench the BSA tryptophan residues. The slope of the straight line of best fit is termed the SternVolmer quenching constant $\left(K_{S V}\right)$. From the data shown in Figure 4(a), $K_{S V^{-}}$-values of $10^{4}$ to $10^{5} \mathrm{M}^{-1}$ have been calculated from a straight-line plot through the linear region at low tannin concentrations (Table 3) and are comparable with $K_{S V^{-}}$values of $10^{4} \mathrm{M}^{-1}$ for tea, coffee and cocoa extracts ${ }^{31}$ and with $6.85 \times 10^{4} \mathrm{M}^{-1}$ for quenching of human serum albumin by epigallocatechin gallate $(\mathrm{EGCg}) .{ }^{50}$ These fluorescence data complement the ITC data by providing additional support to the findings outlined above. The trend in terms of the quenching constants, $K_{S V}$, matches that seen from ITC measurements of the affinity constants, $K_{l}$, with a linear correlation $\left(\mathrm{R}^{2}=0.988\right)$ between $K_{S V}$ and the binding affinity constant, $K_{l}$ (Tables 2 and 3). 
392 However, it should be noted that each of the Stern-Volmer plots is non-linear and

393 curves towards the $y$-axis with higher tannin concentration. This is not unusual in

394 Stern-Volmer plots and provides information on the type of quenching. An upward

395 slope suggests a combination of both static and dynamic quenching in these

396 ellagitannin-BSA systems. ${ }^{42}$ Static quenching is associated with a strong coupling

397 (e.g. formation of a ground-state complex) between interacting molecules, whereas

398 dynamic quenching is associated with weak coupling (e.g. through-space interactions

399 or collisions). ${ }^{42}$ To further confirm that the curvature was indeed due to combined

400 static and dynamic quenching, a modified form of the Stern-Volmer equation was

401 used (see Methods) that is second order with respect to [Q], and this gave as predicted

402 a linear plot (Figure 4(c)).

403

404 Polarity of the Tryptophan Environment. Fluorescence spectra can also yield

405 information on another parameter, i.e. the position of the emission peak maximum

$406\left(\lambda_{\text {em }}\right)$, which can reflect the polarity of the tryptophan environment. ${ }^{50}$ The presence of

407 ellagitannins led to a bathochromic or red-shift in $\lambda_{\mathrm{em}}$ (Table 3), suggesting that the

408 tryptophan environment became more polar upon interaction with ellagitannins. The

409 observed red-shift may suggest a change to the protein's tertiary structure and thus

410 account for the associated increase in the polarity of the tryptophan environment. ${ }^{28}$

411 However, it is difficult to conclude this based solely on the data reported here. A shift

412 to a more polar environment could suggest unfolding of the protein and thus greater

413 solvent exposure or more H-bonding between BSA and the tannins. However, Li et

$414 a l^{30}$ reported a slight blue-shift upon binding of tannic acid (i.e. a mixture of

415 gallotannins containing either a central glucose or quinic acid) to ferritin, whereas 
416 Dobreva et $a l^{28}$ measured a red-shift of $5 \mathrm{~nm}$ when pure pentagalloyl glucose bound 417 with BSA. The magnitude of the red shift appears to be linked to tannin structure as

418 related monomer-dimer pairs showed some similarities: vescalagin and roburin A generated a 8-9 nm shift, pedunculagin and oenothein B a 4-5 nm shift, but gemin A caused the greatest shift in $\lambda_{\mathrm{em}}$ of $10.3 \mathrm{~nm}$. A similarly large red shift of $12 \mathrm{~nm}$ was observed for Trp during EGCg binding to human serum albumin. ${ }^{50}$ Other experiments will be needed to establish the precise reasons for the observed differences in redshifts.

In conclusion, our ITC data suggest that there are two types of binding sites for ellagitannin binding to BSA. A stronger binding site may well be more selective and dependent on tannin flexibility and the presence of free galloyl groups. Indeed, our previous study suggested a link between the number of aromatic residues on the surface of a globular protein, Rubisco, and pentagalloyl glucose binding. ${ }^{28}$ This could explain the relationship between $K_{1}$ and the hydrophobic accessible surface area, ASA_H (Figure 5), i.e. $K_{l}=0.2125 \mathrm{x} \mathrm{e}$ 0.0114ASA_H $\left(\mathrm{R}^{2}=0.965\right)$, and points to the fact that hydrophobic interactions are an important driver for affinity during BSAellagitannin complex formation as supported by positive entropies for 5 of the 6 ellagitannins studied here. -

A second weaker binding site was required to fit our data in order to allow for the binding interactions observed at high tannin:protein molar ratios. This second binding site was associated with high values of $n_{2}$ for all tannins and suggests non-selective adsorption of tannin to the protein surface. Such findings suggest tannin binding to BSA is concentration dependent, where specific binding might dominate at low 
441

442 These studies have shown that ellagitannin binding to BSA increases in strength and

443 affinity for the larger tannins (dimers) compared to their monomer forms. Bond

444 rotational flexibility of the tannin also plays a role by increasing the strength of

445 interaction and number of stronger (possibly hydrogen bonding) binding sites on the

446 protein surface.

447

448

449

450

$451 \quad$ Funding

452 This study was supported by the EU Marie Curie Research Training Network

453 'HealthyHay' (contract: MRTN-CT-2006-035805) and Academy of Finland (grant

454258992 to JPS).

455

456 ACKNOWLEDGMENTS

457 Kristiina Lehtonen is acknowledged for help with ellagitannin purification.

458 


\section{REFERENCES}

(1) Okuda, T.; Ito, H. Tannins of constant structure in medicinal and food plants hydrolysable tannins and polyphenols related to tannins. Molecules 2011, 16, 21912217.

(2) Vrhovsek, U.; Guella, G.; Gasperotti, M.; Pojer, E.; Zancato, M.; Mattivi, F. Clarifying the identity of the main ellagitannins in the fruit of the strawberry, Fragaria vesca and Fragaria ananassa Duch. J. Agric. Food Chem. 2012, 60, 25072516.

(3) Larrosa, M.; Garcia-Conesa, M. T.; Espin, J. C.; Tomas-Barberan, F. A. Ellagitannins, ellagic acid and vascular health. Mol. Aspects Med. 2010, 31, 513-539. (4) Quideau, S.; Genot, E.; Saltel, F.; Douat-Casassus, C.; Delannoy Lopez, D. M. 2012. C-glucosidic ellagitannin compounds for use for altering the supramolecular arrangement of actin and for the treatment of osteoporosis, cancer, bacterial infection and viral infection. International Patent Application WO 2012/113835 A1 (PCT/EP2012/053017),

(5) Quideau, S.; Deffieux, D.; Douat-Casassus, C.; Pouységu, L. Plant polyphenols: chemical properties, biological activities, and synthesis. Angew. Chem. Int. Ed. 2011a, 50, 5099-5104.

(6) Scalbert, A.; Andres-Lacueva, C.; Arita, M.; Kroon, P.; Manach, C.; UrpiSarda, M.; Wishart, D. Databases on food phytochemicals and their health-promoting effects. J. Agric. Food Chem. 2011, 59, 4331-4348.

(7) Rothwell, J. A.; Urpi-Sarda, M.; Boto-Ordonez, M.; Knox, C.; Llorach, R.; Eisner, R.; Cruz, J.; Neveu, V.; Wishart, D.; Manach, C.; Andres-Lacueva, C.; Scalbert, A. Phenol-Explorer 2.0: a major update of the Phenol-Explorer database 
integrating data on polyphenol metabolism and pharmacokinetics in humans and experimental animals. Database 2012, doi:10.1093/database/bas031.

(8) Fotschki, B.; Milala, J.; Jurgonski, A.; Karlińska, E.; Zdunczyk, Z.; Juskiewicz, J.. Strawberry ellagitannins thwarted the positive effects of dietary fructooligosaccharides in rat cecum. J. Agric. Food Chem. 2012, 62, 5871-5880.

(9) Salminen, J. P.; Karonen, M.; Sinkkonen, J. Chemical ecology of tannins: recent developments in tannin chemistry reveal new structures and structure-activity patterns. Chem. Eur. J. 2011, 17, 2806-2816.

(10) Quideau, S.; Douat-Casassus, C.; Delannoy López, D. M.; Di Primo, C.; Chassain, S.; Jacquet, R.; Saltel, F.; Genot, E. Binding of filamentous actin and winding into fibrillar aggregates by the polyphenolic C-glucosidic ellgitannin vescalagin. Angew. Chem. Int. Ed. 2011b, 50, 5099-5104.

(11) Okuda, T. Systematics and health effects of chemically distinct tannins in medicinal plants. Phytochemistry 2005, 66, 2012-2031.

(12) Kashiwada, Y.; Nonaka, G.-I.; Nishioka, I.; Lee, K. J.-H.; Bori, I.; Fukushima, Y.; Bastow, K. F.; Lee, K.-H. Tannins as potent inhibitors of DNA topoisomerase-II in vitro. J. Pharm. Sci. 1993, 82, 487-492.

(13) Quideau, S.; Jourdes, M.; Saucier, C.; Glories, Y.; Pardon, P.; Baudry, C. DNA topoisomerase inhibitor acutissimin A and other flavano-ellagitannins in red wine. Angew. Chem. Int. Ed. 2003, 42, 6012-6014.

(14) Auzanneau, C.; Montaudon, D.; Jacquet, R.; Puyo, S.; Pouységu, L.; Deffieux, D.; Elkaoukabi-Chaibi, A.; De Giorgi, F.; Ichas, F.; Quideau, S.; Pourquier, P. The polyphenolic ellagitannin vescalagin acts as a preferential catalytic inhibitor of the $\alpha$ isoform of human DNA topoisomerase II. Mol. Pharmacol. 2012, 82, 124-141. 
(15) Kiss, A. K.; Granica, S.; Stolarczyk, M.; Melzig, M. F. Epigenetic modulation of mechanisms involved in inflammation: influence of selected polyphenolic substances on histone acetylation state. Food Chem. 2012, 131, 1015-1020.
(16) Cala, O.; Dufourc, E.J.; Fouquet, E.; Manigand, C.; Laguerre, M.; Pianet, I.

The colloidal state of tannins impacts the nature of their interaction with proteins: the

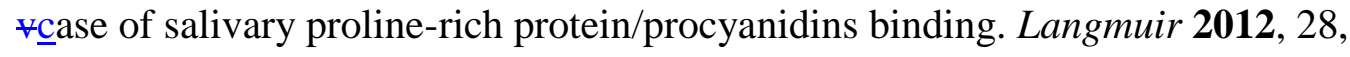
$17410-17418$.

(17) Fraga, C. G.; Galleano, M.; Verstraeten S. V.; Oteiza, P. I. Basic biochemical mechanisms behind the health benefits of polyphenols. Mol. Aspects Med. 2010, 31, $435-445$.

(18) Bacon, J.R.; Rhodes, M.J.C. Binding affinity of hydrolyzable tannins to parotid saliva and to proline-rich proteins derived from it. J. Agric. Food Chem., 2000, $48,838-843$.

(19) Frazier, R. A.; Deaville, E. R.; Green, R. J.; Willoughby, I.; Stringano, E.; Plant, J.; Mueller-Harvey, I. Interactions of tea tannins and condensed tannins with proteins. J. Pharm. Biomed. Anal. 2010, 51, 490-495.

(20) Canon, F.; Ballivian, R.; Chirot, F.; Antoine, R.; Sarni-Manchado, P.; Lemoine, J.; Dugourd, P. Folding of a salivary intrinsically disordered protein upon binding to tannins. J. Am. Chem. Soc. 2011, 133, 7847-7852.

(21) Delannoy, D.; Deffieux, D.; Di Primo, C.; Quideau S. Study in real time of polyphenol-protein interactions by surface plasmon resonance. Polyphenols Commun. 2012, 1, 59-60.

(22) Simon, C.; Barathieu, K.; Laguerre, M.; Schmitter, J.-M.; Fouquet, E.; Pianet, I.; Dufourc, E. J. Three-dimensional structure and dynamics of wine tannin- 
saliva protein complexes. A multitechnique approach. Biochemistry 2003, 42, 1038510395.

(23) Hagerman, A.E. Fifty years of polyphenol-protein complexes. Chapter 3 in Recent Advances in Polyphenol Research. Cheynier, V.; Sarni-Manchado, P.; Quideau, S., Eds; John Wiley \& Sons Ltd, Oxford, UK. 2012, 3, 71-97.

(24) Deaville, E. R.; Green, R. J.; Mueller-Harvey, I.; Willoughby, I.; Frazier, R. A. Hydrolysable tannin structures influence relative binding strengths to globular or random coil proteins. J. Agric. Food Chem. 2007, 55, 4554-4561.

(25) Hagerman, A. E; Butler, L. G. The specificity of proanthocyanidin-protein interactions. J. Biol. Chem. 1981, 256, 4494-4497.

(26) Bazylko, A.; Kiss, K. K.; Kowalski, J. High-performance thin-layer chromatography method for quantitative determination of oenothein B and quercetin glucuronide in aqueous extract of Epilobii angustifoliii herba. J. Chromatogr. A 2007, $1173,146-150$.

(27) Giner-Chavez, B-I.; Van Soest, P.J.; Robertson, J.B.; Lascano, C.; Pell, A.N. Comparison of the Precipitation of Alfalfa Leaf Protein and Bovine Serum Albumin by Tannins in the Radial Diffusion Method. J. Sci. Food Agric. 1997, 74, 513-523. (28) Dobreva, M. A.; Frazier, R. A.; Mueller-Harvey, I.; Clifton, L. A.; Gea A.; Green, R. J. Binding of pentagalloyl glucose to two globular proteins occurs via multiple surface sites. Biomacromolecules 2011, 12, 710-715.

(29) Hofmann, T.; Glabasnia, A.; Schwarz, B.; Wisman, K. N.; Gangwer, K. A.; Hagerman, A. E. Protein binding and astringent taste of a polymeric procyanidin, 1,2,3,4,6-penta-O-galloyl-beta-D-glucopyranose, castalagin, and grandinin. J. Agric. Food Chem. 2006, 54, 9503-9509. 
(30) Li, M.; Jia, X.; Yang, J.; Deng, J.; Zhao, G. Effect of tannic acid on properties of soybean (Glycine max) seed ferritin: A model for interaction between naturallyoccurring components in foodstuffs. Food Chem. 2012, 133, 410-415.

(31) Stojadinovic, M.; Radosavljevic, J.; Ognjenovic, J.; Vesic, J.; Prodic, I.; Stanic-Vucinic, D.; Cirkovic Velickovic T.. Binding affinity between dietary polyphenols and $\beta$-lactoglobulin negatively correlates with the protein susceptibility to digestion and total antioxidant activity of complexes formed. Food Chem. 2013, $136,1263-1271$.

(32) Dufour C.; Dangles O. Flavonoid-serum albumin complexation: determination of binding constants and binding sites by fluorescence spectroscopy. Biochim. Biophys. Acta 2005, 1721, 164-173.

(33) Skrt, M.; Benedik, E.; Podlipnik, C.; Ulrih, N. P. Interactions of different polyphenols with bovine serum albumin using fluorescence quenching and molecular docking. Food Chem. 2012, 135, 2418-2424.

(34) Li, M.; Hagerman, A.E. Interactions between plasma proteins and naturally occurring polyphenols. Curr. Drug Metab. 2013, 14, 432-445.

(35) Zhang, Y.; Shi, S.; Chen, X.; Zhang, W.; Huang, K.; Peng, M. Investigation on the interaction between Ilaprazole and bovine serum albumin without or with different C-ring flavonoids from the viewpoint of food-drug interference. J. Agric. Food Chem. 2011, 59, 8499-8506.

(36) Huang, B.X.; Kim, H-H.; Dass, C. Probing three-dimensional structure of bovine serum albumin by chemical cross-linking and mass spectrometry. J. Am. Soc. Mass Spectrom. 2004, 15, 1237-1247. 
(37) Moilanen, J.; Salminen, J.-P. Ecologically neglected tannins and their biologically relevant activity: chemical structures of plant ellagitannins reveal their in vitro oxidative activity at high $\mathrm{pH}$. Chemoecology 2008, 18, 73-83.

(38) O’Brien, R.; Ladbury, J. E.; Chowdhry, B. Z. Isothermal titration calorimetry of biomolecules. In: Protein-Ligand Interactions: Hydrodynamics and Calorimetry; Harding, S. E.; Chowdhry, B. Z. (Eds.); Oxford University Press: Oxford, U.K., 2001; pp. 263-286.

(39) Frazier, R. A.; Papadopoulou, A.; Mueller-Harvey, I.; Kissoon, D.; Green, R. J. Probing protein-tannin interactions by isothermal titration microcalorimetry. J. Agric. Food Chem. 2003, 51, 5189-5195.

(40) Freire, E.; Mayorga, O .L.; Straume, M. Isothermal titration calorimetry. Anal. Chem. 1990, 62, A950-A959.

(41) Chi, Z.; Liu, R.; Zhang, H. Noncovalent interaction of oxytetracycline with the enzyme trypsin. Biomacromolecules 2010, 11, 2454-2459.

(42) Lakowicz, J. R. Principles of Fluorescence Spectroscopy; 2nd ed.; Kluwer Academic/Plenum Publishers: New York, 1999; p 698.

(43) Vivas, N.; Laguerre, M.; Pianet de Boissel, I.; Vivas de Gaulejac, N.; Nonier, M.-F. Conformational interpretation of vescalagin and castalagin physicochemical properties. J. Agric. Food Chem. 2004, 52, 2073-2078.

(44) McManus, J. P.; Davis, K. G.; Beart, J. E.; Gaffney, S. H.; Lilley, T. H.; Haslam, E. Polyphenol interactions. Part I. Introduction: some observations on the reversible complexation of polyphenols with proteins and polysaccharides. J. Chem. Soc. Perkin Trans. 2, 1985, 1429-1438. 
602

603

604

605

606

607

608

609

610

611

612

613

614

615

616

617

618

619

620

621

622

623

624

625

626

(45) Charlton, A.J.; Baxter, N.J; Khan, M.L; Moir, A.J.G.; Haslam, E.; Davies,

A.P.; Williamson, M.P. Polyphenol/peptide binding and precipitation. J. Agric. Food

Chem. 2002, 50, 1593-1601.

(46) Mishra, B.; Barik, A.; Priyadarsini, K. I.; Mohan, H. Fluorescence

spectroscopy studies on binding of a flavanoid antioxidant quercetin to serum albumins. J. Chem. Sci. 2005, 117, 641-647.

(47) Tian, J.; Liu, J.; Tina X.; Hu, Z.; Chen, X. G. Study of the interaction of kaempferol with bovine serum albumin. J. Mol. Struct. 2004, 691, 197-202.

(48) Tian, J.; Liu, J.; Hu, Z.; Chen, X. G. Interaction of wogonin with bovine serum albumin. Bioorg. Med. Chem. 2005, 13, 4124-4129.

(49) Lorrain, B.; Dangles, O.; Genot, C.; Dufour, C. Chemical modeling of hemeinduced lipid oxidation in gastric conditions and inhibition by dietary polyphenols. $J$. Agric. Food Chem. 2010, 58, 676-683.

(50) Maiti, T. K.; Gosh, K. S.; Dsagupta, S. Interaction of (-)-epigallocatchin-3gallate with human serum albumin: fluorescence, Fourier transform infrared, circular dichroism, and docking studies. Protein Struct. Funct. Genet. 2006, 64, 355-362.

(51) Zhang, G.; Wang, L.; Pan, J. Probing the binding of the flavonoid diosmetin to human serum albumin by multispectroscopic techniques. J. Agric. Food Chem. 2012, 60, 2721-2729.

(52) Xiao, J.; Zhao, Y.; Wang, H.; Yuan, Y.; Yang, F.; Zhang, C.; Yamamoto, K.

Noncovalent interaction of dietary polyphenols with common human plasma proteins. J. Agric. Food Chem. 2011, 59, 10747-10754.

(53) Zhu, M. J.; Phillipson, J. D.; Greengrass, P. M.; Bowery, N. E.; Cai, Y. Plant polyphenols: biologically active molecules or non-selective binders to protein?

Phytochemistry 1997, 44, 441-447. 
627 (54) Chandler, D. Interfaces and the driving force of hydrophobic assembly.

$628 \quad$ Nature 2005, 437, 640-647.

629 (55) Butler, L. G.; Riedl, D. J.; Lebryk, D. G.; Blytt, H. J. Interaction of proteins

630 with sorghum tannin: mechanisms, specificity and significance. J. Am. Oil Chem. Soc.

$631 \quad \mathbf{1 9 8 4}, 61,916-920$.

632 
Figure 1. Structures of ellagitannins and pentagalloyl glucose.

636

637 Figure 2. ITC binding isotherms for the interaction of bovine serum albumin with the monomer-dimer pair of (a) vescalagin and (b) roburin A. Data were fitted assuming a model for two-independent binding sites and values obtained from these fits are given in Table 2.

641

Figure 3. ITC binding isotherms for the interaction of bovine serum albumin with the monomer-dimer pairs of (a) pendunculagin, (b) oenothein B and (c) gemin A. Data were fitted assuming a model for two-independent binding sites and values obtained from these fits are given in Table 2.

Figure 4. Fluorescence quenching of tryptophans in BSA by vescalagin, roburin A, pedunculagin, oenothein B and gemin A plotted as: (a) ratio of initial fluorescence intensity to total fluorescence intensity versus ellagitannin concentration; (b) ratio of initial fluorescence intensity to total fluorescence intensity versus ellagitannin monomer concentration; and as a (c) modified Stern-Volmer plot to reflect

652 contributions from both static and dynamic quenching (data are shown for vescalagin, 653 roburin A, pedunculagin and oenothein B only).

654 hydrophobic surface area (ASA_H). 
Table 1: Modeling of minimized energies and associated numbers of conformers, accessible hydrophobic surface area (ASA_H) and accessible surface area (ASA).

\begin{tabular}{lccccc}
\hline \multicolumn{1}{c}{ Compound } & $\begin{array}{c}\text { Molecular } \\
\text { weight } \\
\text { (Daltons) }\end{array}$ & $\begin{array}{c}\text { Energies of } \\
\text { conformers } \\
\text { (kcal/mol) }\end{array}$ & $\begin{array}{c}\text { Number of } \\
\text { conformers }\end{array}$ & $\begin{array}{c}\text { ASA_H } \\
\mathbf{n m}^{\mathbf{2}}\end{array}$ & $\begin{array}{c}\mathbf{A S A} \\
\mathbf{n m}^{\mathbf{2}}\end{array}$ \\
\hline $\begin{array}{l}\text { Vescalagin } \\
\text { Roburin A }\end{array}$ & 935 & 229 to 236 & 18 & 253 & 914 \\
& 1843 & 406 to 412 & 4 & 407 & 1587 \\
Pedunculagin & 769 & 158 to 164 & 4 & 293 & 867 \\
Oenothein B & 1569 & 278 to 280 & 2 & 524 & 1485 \\
& 927 & 110 to 116 & 21 & 373 & 1041 \\
Pentagalloyl glucose & 1841 & 323 & 1 & 574 & 1748 \\
Gemin A & & & & & \\
\hline
\end{tabular}


Table 2: Estimated thermodynamic binding parameters for the interaction between five ellagitannins and pentagalloyl glucose ${ }^{\mathrm{b}}$ with bovine serum albumin at $\mathrm{pH}$ 6. $\Delta H_{1}$ and $\Delta H_{2}$ are the enthalpies, and $K_{1}$ and $K_{2}$ are the equilibrium binding constants for two binding sites.

\begin{tabular}{ccccccc}
\hline & Vescalagin & Roburin A & Pedunculagin & Oenothein B & $\begin{array}{c}\text { Pentagalloyl } \\
\text { glucose }\end{array}$ & Gemin A \\
& $2.5 \pm 0.5^{\mathrm{a}}$ & 1.9 & 2.1 & 4.1 & 26 & 13 \\
\hline$n_{1}$ & $5.2( \pm 2.3) \times 10^{4}$ & $2.3 \times 10^{5}$ & $4.2 \times 10^{4}$ & $6.5 \times 10^{5}$ & $1.8 \times 10^{5}$ & $1.8 \times 10^{6}$ \\
$K_{1}\left(\mathrm{M}^{-1}\right)$ & $-7.5 \pm 1.0$ & -15.3 & -10.9 & -20.6 & -29 & -47.4 \\
$\Delta H_{1}\left(\mathrm{~kJ} \mathrm{~mol}^{-1}\right)$ & 65.1 & 33.2 & 51.9 & 42.2 & 3.3 & -39.3 \\
$\Delta S_{1}\left(\mathrm{~J} \mathrm{~mol}^{-1} \mathrm{~K}^{-1}\right)$ & $30 \pm 4$ & 16 & 30 & 20 & 26 & 25 \\
$n_{2}$ & $1.1( \pm 0.07) \times 10^{3}$ & $1.8 \times 10^{4}$ & $1.1 \times 10^{3}$ & $3.3 \times 10^{4}$ & $8 \times 10^{2}$ & $6.3 \times 10^{4}$ \\
$K_{2}\left(\mathrm{M}^{-1}\right)$ & $-6.7 \pm 1.3$ & -8.17 & -5.1 & -10.3 & -29 & -11.4 \\
$\Delta H_{2}\left(\mathrm{~kJ} \mathrm{~mol}^{-1}\right)$ & 35.7 & 54.0 & 41.1 & 51.9 & -41.7 & 53.6 \\
$\Delta S_{2}\left(\mathrm{~J} \mathrm{~mol}^{-1} \mathrm{~K}^{-1}\right)$ & $0.65 \pm 0.16$ & 0.87 & 1.99 & 8.07 & & 3.29
\end{tabular}

\footnotetext{
${ }^{\mathrm{a}} \pm$ standard deviation, $\mathrm{n}=3 .{ }^{\mathrm{b}}$ Data are included for comparison (Dobreva et al. $^{28}$ ).
} 
Table 3: Estimated quenching parameters for the interaction of ellagitannins with bovine serum albumin $(n=3)$.

\begin{tabular}{llllll}
\hline Fluorescence parameters & Vescalagin & Roburin A & Pedunculagin & Oenothein B & Gemin A \\
\hline$K_{S V}\left(\mathrm{M}^{-1}\right)$ & $0.42( \pm 0.07) \times 10^{5}$ & $1.03( \pm 0.07) \times 10^{5}$ & $0.53( \pm 0.1) \times 10^{5}$ & $1.44( \pm 0.28) \times 10^{5}$ & $3.13( \pm 0.06) \times 10^{5}$ \\
& & & & & \\
Bathochromic (red)-shift of & $8.7 \pm 0.4$ & $8.7 \pm 0.4$ & $4.7 \pm 0.9$ & $4.0 \pm 1.0$ & $10.3 \pm 0.4$ \\
$\lambda_{\max }(\mathrm{nm})$ & & & & & \\
\hline
\end{tabular}



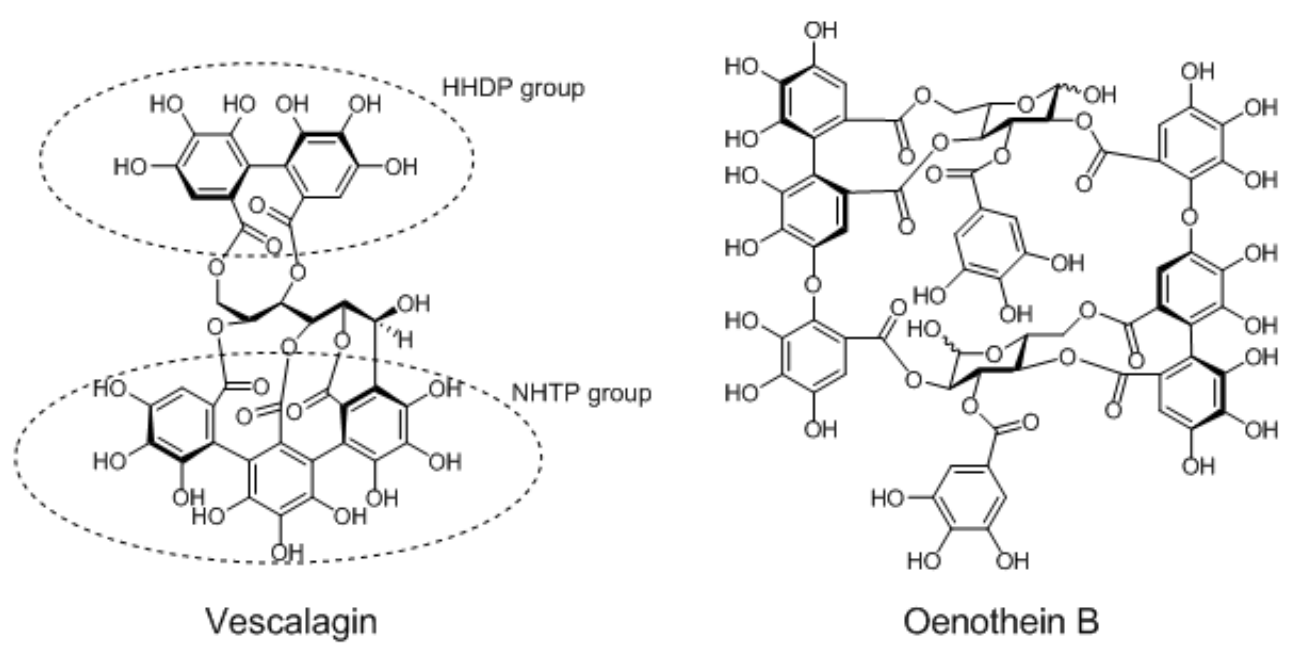

Oenothein B

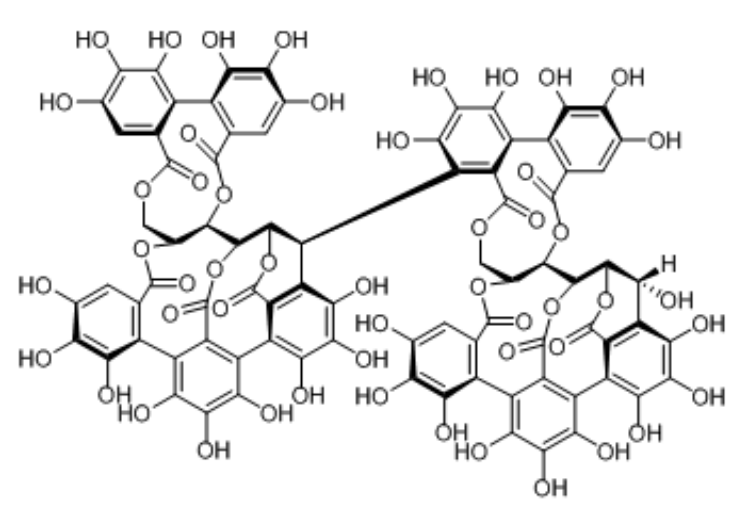

Roburin A

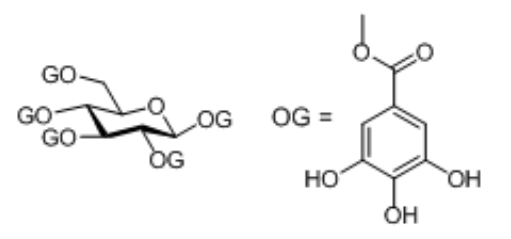

Pentagalloyl glucose

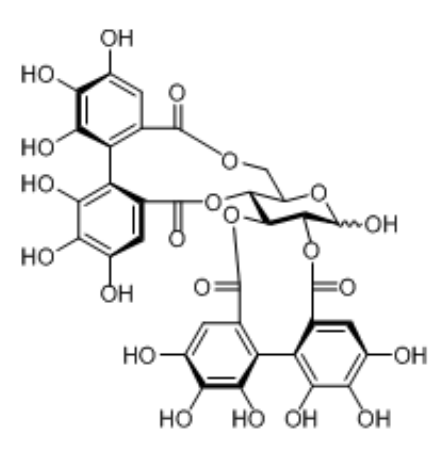

Pedunculagin

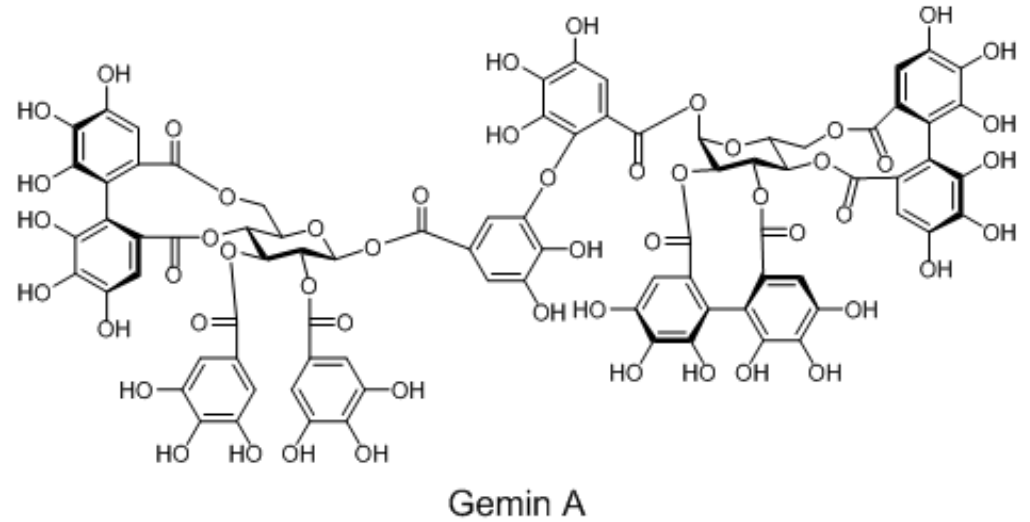

Gemin A

\section{Figure 1}




\section{FIGURE 2}
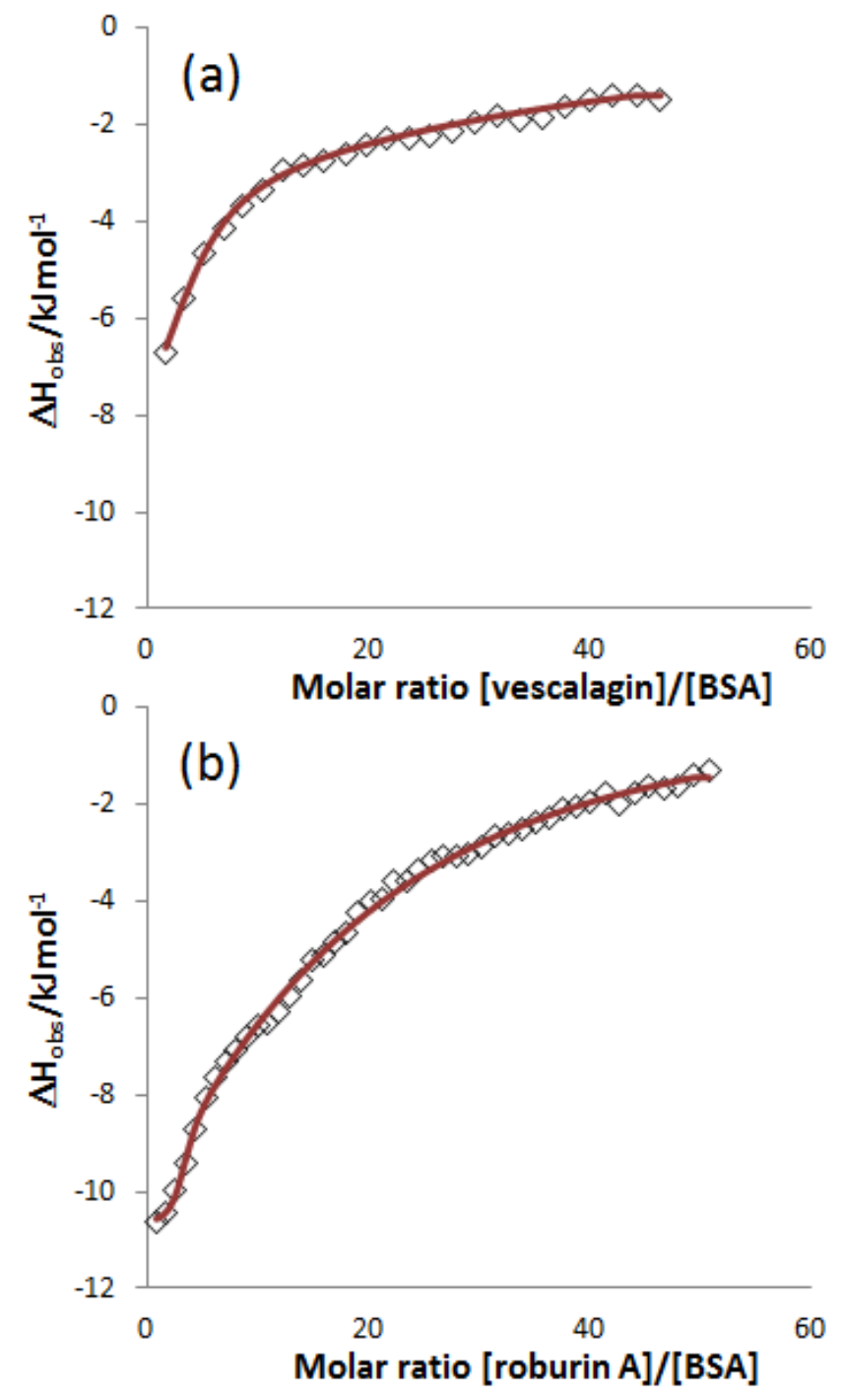

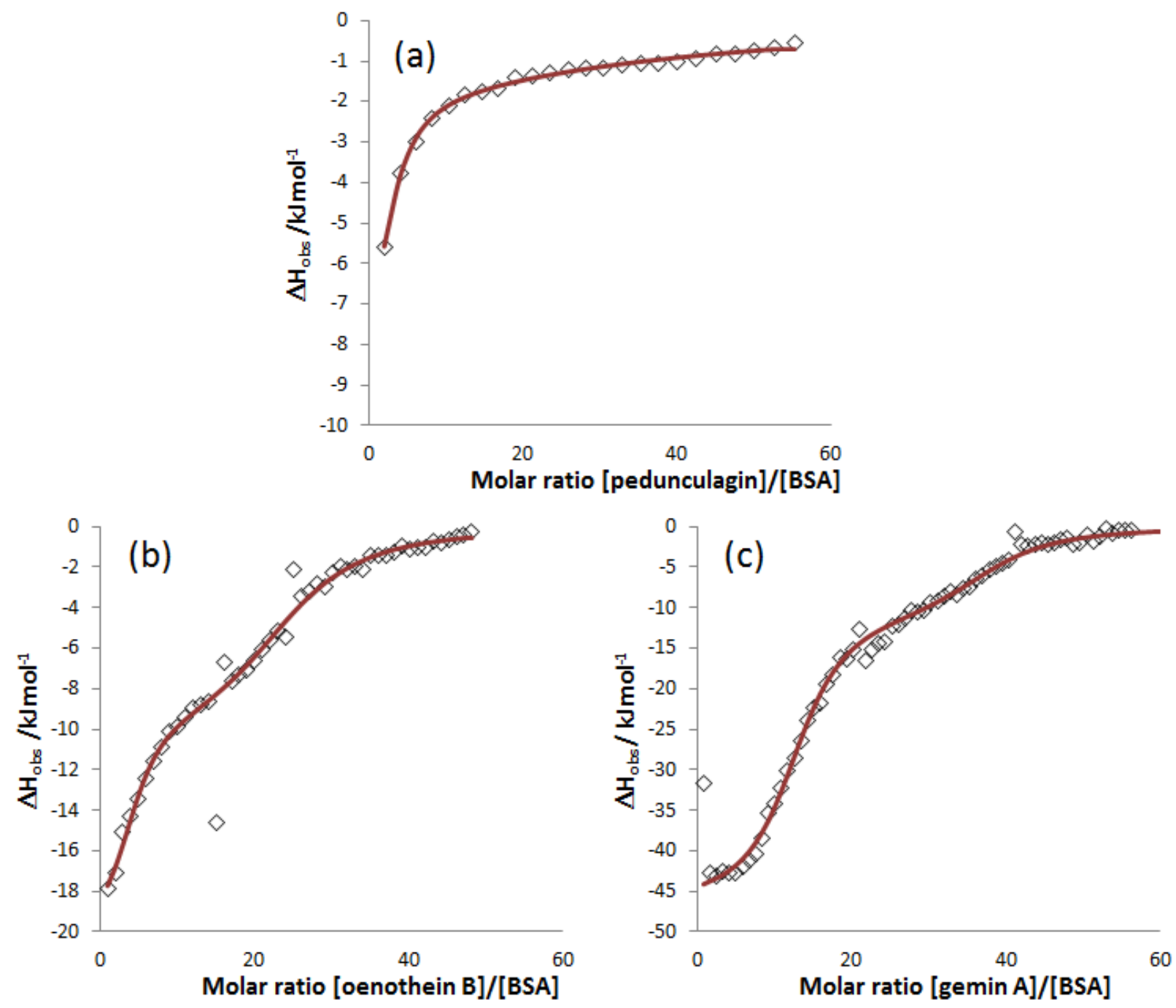

\section{FIGURE 3}



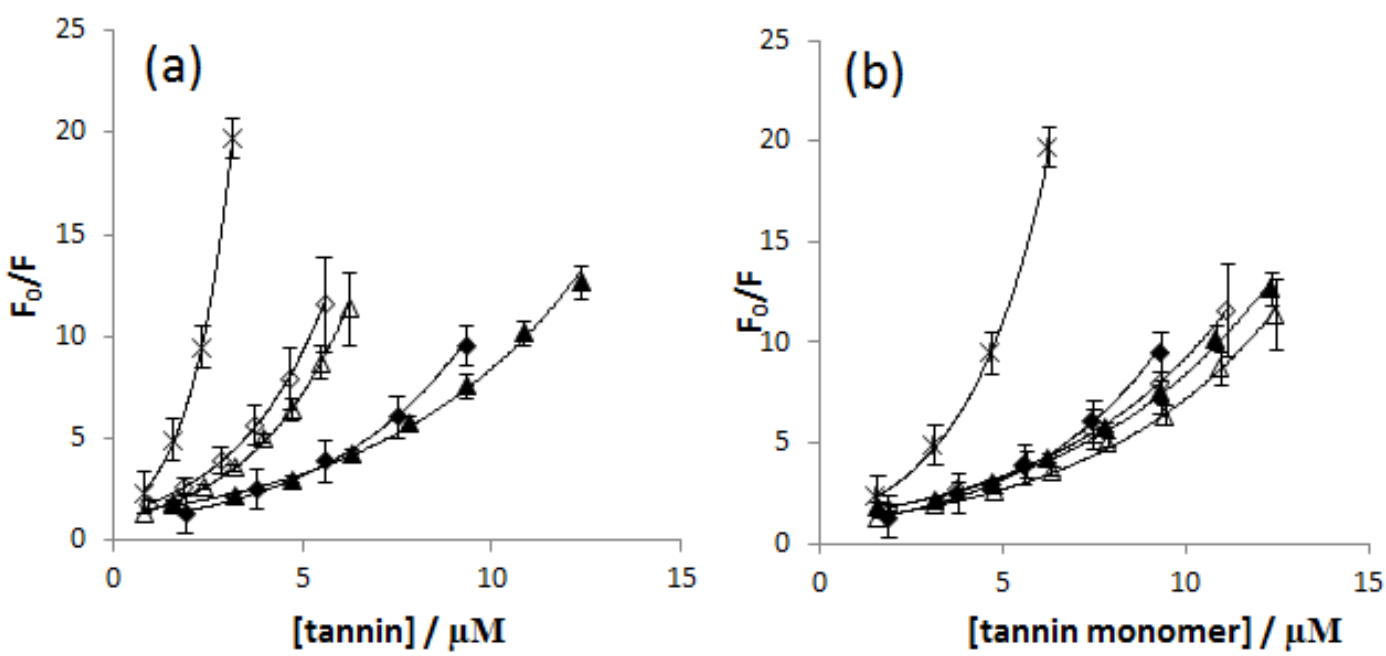

\section{FIGURE 4}

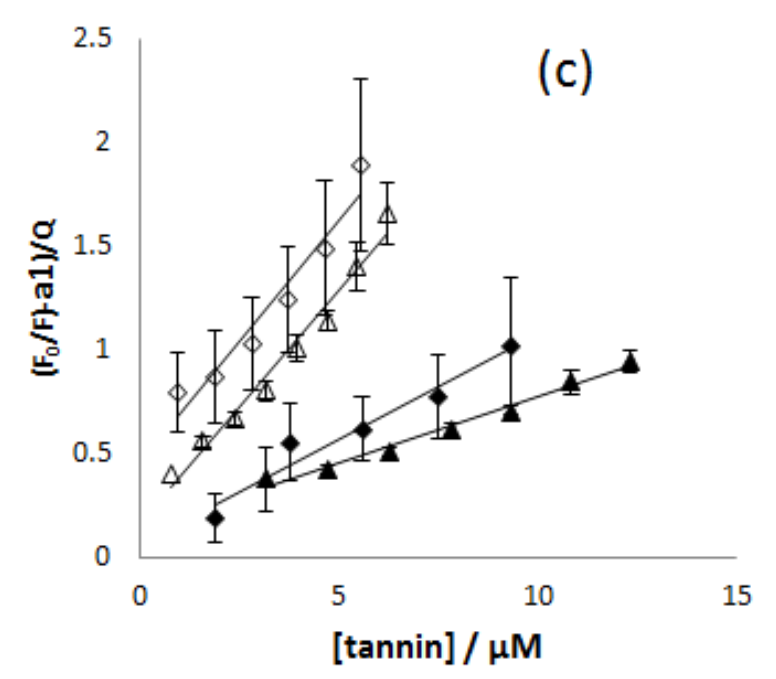




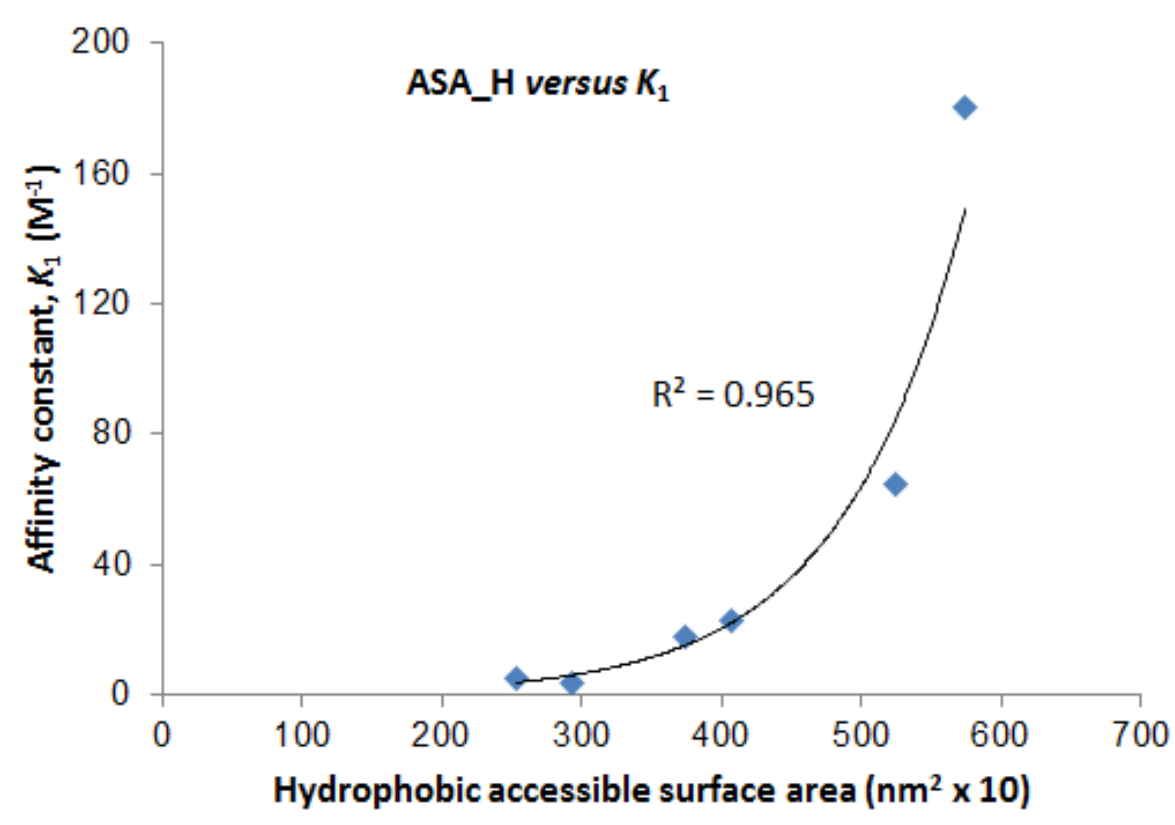

\section{FIGURE 5}




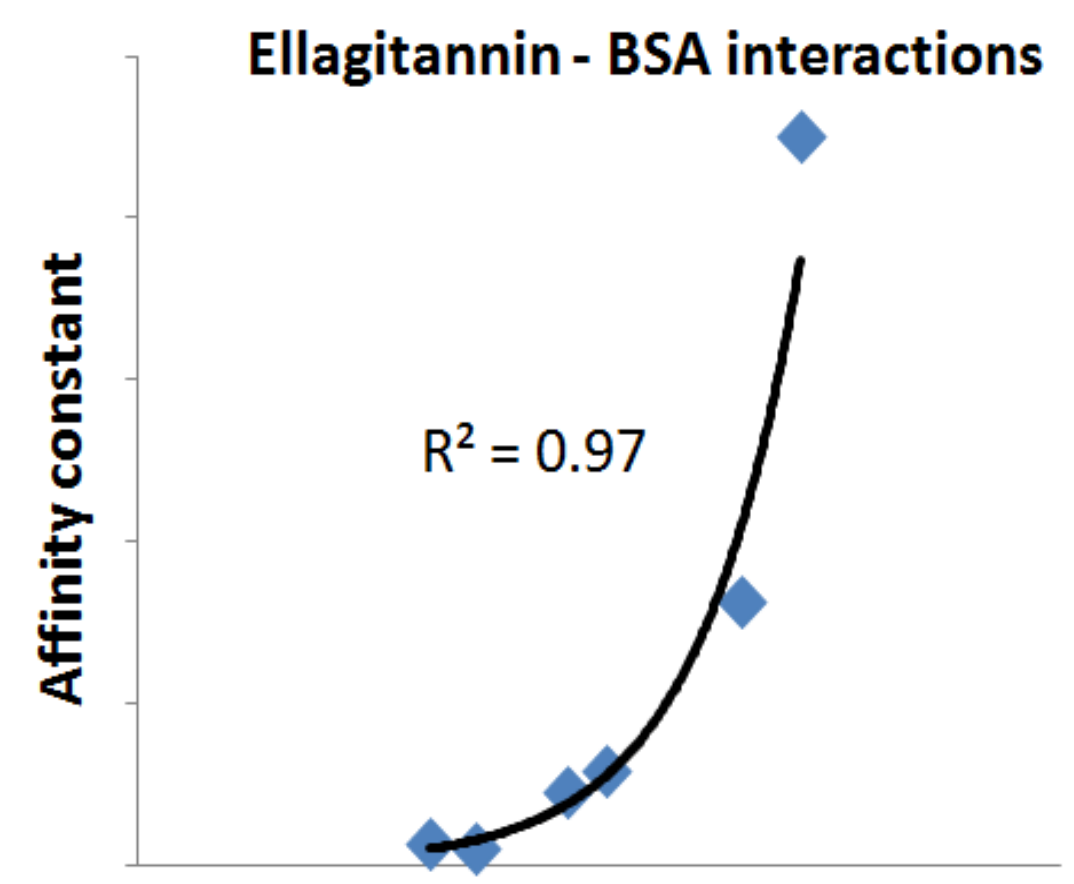

Hydrophobic accessible surface area of ellagitannins 